\title{
Constraints on the neutrino mass and mass hierarchy from cosmological observations
}

\author{
Qing-Guo Huang ${ }^{1,2, a}$, Ke Wang ${ }^{1, b}$, Sai Wang ${ }^{1, c}$ \\ ${ }^{1}$ Key Laboratory of Theoretical Physics, Institute of Theoretical Physics, Chinese Academy of Sciences, Beijing 100190, China \\ ${ }^{2}$ School of Physical Sciences, University of Chinese Academy of Sciences, No. 19A Yuquan Road, Beijing 100049, China
}

Received: 24 March 2016 / Accepted: 20 August 2016 / Published online: 6 September 2016

(C) The Author(s) 2016. This article is published with open access at Springerlink.com

\begin{abstract}
Considering the mass splitting between three active neutrinos, we represent the new constraints on the sum of neutrino mass $\sum m_{v}$ by updating the anisotropic analysis of the Baryon Acoustic Oscillation (BAO) scale in the CMASS and LOWZ galaxy samples from Data Release 12 of the SDSS-III Baryon Oscillation Spectroscopic Survey (BOSS DR12). Combining the BAO data of 6dFGS, MGS, LOWZ and CMASS with Planck 2015 data of temperature anisotropy and polarizations of the Cosmic Microwave Background (CMB), we find that the $95 \%$ C.L. upper bounds on $\sum m_{v}$ refer to $\sum m_{v, \mathrm{NH}}<0.18 \mathrm{eV}$ for the normal hierarchy $(\mathrm{NH}), \sum m_{\nu, \mathrm{IH}}<0.20 \mathrm{eV}$ for the inverted hierarchy (IH) and $\sum m_{v, \mathrm{DH}}<0.15 \mathrm{eV}$ for the degenerate hierarchy (DH), respectively, and the normal hierarchy is slightly preferred over the inverted one $\left(\Delta \chi^{2} \equiv \chi_{\mathrm{NH}}^{2}-\chi_{\mathrm{IH}}^{2} \simeq-3.4\right)$. In addition, the additional relativistic degrees of freedom and massive sterile neutrinos are neither favored at present.
\end{abstract}

\section{Introduction}

The phenomena of neutrino oscillation imply that there are mass splittings between the three active neutrinos (see [1] for a review). Currently only two independent mass squared differences have been determined by neutrino oscillation experiments. Regardless of experimental uncertainties, they are given by [2]

$$
\begin{aligned}
& \Delta m_{21}^{2} \equiv m_{2}^{2}-m_{1}^{2}=7.5 \times 10^{-5} \mathrm{eV}^{2}, \\
& \left|\Delta m_{31}^{2}\right| \equiv\left|m_{3}^{2}-m_{1}^{2}\right|=2.5 \times 10^{-3} \mathrm{eV}^{2} .
\end{aligned}
$$

Thus we have two possible mass hierarchies, namely, a normal hierarchy (NH, $m_{1}<m_{2}<m_{3}$ ) and an inverted hierarchy $\left(\mathrm{IH}, m_{3}<m_{1}<m_{2}\right)$. Here $m_{i}(i=1,2,3)$ denote

\footnotetext{
a e-mail: huangqg@itp.ac.cn

b e-mail: wangke@itp.ac.cn

c e-mail: wangsai@itp.ac.cn
}

the mass eigenvalues of the three neutrinos. The minimum sums of the neutrino masses are $0.06 \mathrm{eV}$ for $\mathrm{NH}$ and 0.10 $\mathrm{eV}$ for $\mathrm{IH}$. Up to now, the absolute neutrino mass and mass hierarchy are still unknown.

Cosmology provides possibilities to measure the neutrino mass or the sum of the neutrino mass $\sum m_{v}$ [3-20]. Massive neutrinos are initially relativistic and become non-relativistic today. They can impact on the cosmic expansion since they evolve differently from pure radiations and pure cold dark matter. They can influence the evolution of cosmological perturbations at early times and affect the CMB temperature anisotropies via the early-time Integrated Sachs-Wolfe (ISW) effect [14]. In addition, relativistic neutrinos suppress the clustering of matter and then modify the growth of structure. Thus one might extract useful signals of cosmic neutrinos from cosmological observations such as the matter clustering and the anisotropies and polarizations of the Cosmic Microwave Background (CMB), etc.

The Planck collaboration [17] gave the $95 \%$ C.L. upper bounds on the total mass of three active neutrinos by assuming a degenerate hierarchy ( $\mathrm{DH}$, where $\left.m_{1}=m_{2}=m_{3}\right)$ regardless of the mass splitting. The Planck TT + lowP constraint is $\sum m_{v}<0.72 \mathrm{eV}$ and the Planck TT, TE, EE + lowP constraint is $\sum m_{v}<0.49 \mathrm{eV}$ for the $\nu_{\mathrm{DH}} \Lambda \mathrm{CDM}$ model. Here TT denotes the power spectrum of CMB temperature, $\mathrm{EE}$ denotes the power spectrum of $\mathrm{CMB} \mathrm{E}$-mode, and TE denotes the CMB temperature and E-mode cross correlation in the Planck 2015 data. "lowP" stands for Planck 2015 low- $\ell$ temperature-polarization data. Further adding the Planck 2015 CMB lensing data [21], the constraints are slightly changed to $\sum m_{v}<0.68 \mathrm{eV}$ and $\sum m_{v}<0.59 \mathrm{eV}$ for two data combinations, respectively. However, by contrast, adding the Baryon Acoustic Oscillation (BAO) data including 6dFGS [22], MGS [23], BOSS DR11 CMASS [24] and LOWZ [24] can significantly improve the constraints to $\sum m_{v}<0.21 \mathrm{eV}$ and $\sum m_{v}<0.17 \mathrm{eV}$, respectively. The 
reason is that the BAO data can significantly break the acoustic scale degeneracy.

Recently the BAO distance scale measurements were updated via an anisotropic analysis of the BAO scale in the correlation function [25] and power spectrum [26] of the CMASS and LOWZ galaxy samples from Data Release 12 of the SDSS-III Baryon Oscillation Spectroscopic Survey (BOSS DR12). The total volume probed in DR12 has a $10 \%$ increment from DR11 and the experimental uncertainty has been reduced correspondingly. Thus in this paper we update the constraints on the total mass of three active neutrinos by using BOSS DR12 CMASS and LOWZ data, which are combined with other cosmological observations such as Planck 2015 CMB data. In this paper, we also consider the mass splitting between three neutrinos implied by the neutrino oscillations between three generations. We estimate whether the current datasets can distinguish the neutrino mass hierarchy. In addition, we also update the constraints on additional relativistic degrees of freedom $\Delta N_{\text {eff }} \equiv N_{\text {eff }}-3.046$ and massive sterile neutrinos $m_{v, \text { sterile }}^{\text {eff }}$.

The rest of the paper is arranged as follows. In Sect. 2, we reveal our methodology and cosmological datasets used in this paper. In Sect. 3, we demonstrate our constraints on the sum of the neutrino mass, additional relativistic degrees of freedom, and massive sterile neutrinos, respectively. Our conclusions are listed in Sect. 4.

\section{Data and method}

The recent distance measurements from the anisotropic analysis of the BAO scale in the correlation function [25] and power spectrum [26] of CMASS and LOWZ galaxy samples from BOSS DR 12 are listed in Table 1.

Only the consensus values [26] are listed, which are used in this paper. Here $z$ denotes the effective redshift for CMASS and LOWZ samples, respectively, $H(z)$ and $D_{A}(z)$ are the Hubble parameter and angular diameter distance at redshift $z$, respectively, and $r_{d}$ is the comoving sound horizon at the redshift of the baryon drag epoch. In addition, $\rho_{D_{A}, H}$ stands for the normalized correlation between $D_{A}(z)$ and $H(z)$.

In this paper, we combine the BAO data including 6dFGS [22], MGS [23], BOSS DR12 CMASS [26], and LOWZ [26] with Planck 2015 likelihoods [27] of CMB temperature and

Table 1 The distance measurement from the anisotropic analysis of the BAO scale in the CMASS and LOWZ galaxy samples released by SDSS-III BOSS DR12. Here we list the consensus values [26]

\begin{tabular}{lllll}
\hline$z$ & BOSS DR12 & $H(z) r_{d}\left(10^{3} \mathrm{~km} \cdot s^{-1}\right)$ & $D_{A}(z) / r_{d}$ & $\rho_{D_{A}, H}$ \\
\hline 0.32 & LOWZ & $11.64 \pm 0.70$ & $6.76 \pm 0.15$ & 0.35 \\
0.57 & CMASS & $14.66 \pm 0.42$ & $9.47 \pm 0.13$ & 0.54 \\
\hline
\end{tabular}

polarizations as well as CMB lensing. In fact, we employ two combinations of observational data, namely Planck TT, TE, $\mathrm{EE}+$ lowP $+\mathrm{BAO}$ and Planck $\mathrm{TT}+$ lowP + lensing + BAO . The latter one is expected to give conservative constraints on the neutrino sectors, while the former one gives more severe constraints. In the base $\Lambda \mathrm{CDM}$ model, the estimates of the amplitude of the fluctuation spectrum inferred from some astrophysical data such as weak lensing (WL) $[28,29]$, redshift space distortion (RSD) [30], and Planck cluster counts [31] is lower than the value inferred from the Planck CMB data. For consistency, we do not take these astrophysical data into consideration in this paper. We neither consider the direct measurements of cosmic expansion, since there are certain debates on the $H_{0}$ data [32-34]. In addition, we do not use the data of supernovae of type Ia (SNe Ia), since the apparent magnitudes of $\mathrm{SNe}$ are insensitive to $\sum m_{v}$.

In the $\Lambda$ CDM model, there are six base cosmological parameters, which are denoted by $\left\{\omega_{b}, \omega_{c}, 100 \theta_{\mathrm{MC}}, \tau, n_{s}\right.$, $\left.\ln \left(10^{10} A_{s}\right)\right\}$. Here $\omega_{b}$ is the physical density of baryons today and $\omega_{c}$ is the physical density of cold dark matter today. $\theta_{\mathrm{MC}}$ is the ratio between the sound horizon and the angular diameter distance at the decoupling epoch. $\tau$ is the Thomson scatter optical depth due to reionization. $n_{s}$ is the scalar spectrum index and $A_{s}$ is the amplitude of the power spectrum of primordial curvature perturbations at the pivot scale $k_{p}=0.05$ $\mathrm{Mpc}^{-1}$.

Because both the physics of $\mathrm{CMB}$ and of $\mathrm{BAO}$ are well understood and hence the systematic uncertainties are under control, we only adopt CMB and BAO datasets and expect to get some conservative constraints on the neutrino mass. Here we also want to stress that our results are based on the assumption that our Universe is spatially flat.

To constrain the neutrino sectors, we refer to the Markov Chain Monte Carlo sampler (CosmoMC) [35] in the $v \Lambda$ CDM model. By considering the mass splitting in Eqs. (1) and (2), we can express the neutrino mass spectrum by two independent mass squared differences and one minimum mass eigenvalue $m_{v, \min }$. The neutrino mass spectrum is

$$
\left(m_{1}, m_{2}, m_{3}\right)=\left(m_{1}, \sqrt{m_{1}^{2}+\Delta m_{21}^{2}}, \sqrt{m_{1}^{2}+\left|\Delta m_{31}^{2}\right|}\right)
$$

and $m_{v, \min }=m_{1}$ for $\mathrm{NH}$, and

$$
\left(m_{1}, m_{2}, m_{3}\right)=\left(\sqrt{m_{3}^{2}+\left|\Delta m_{31}^{2}\right|}, \sqrt{m_{3}^{2}+\left|\Delta m_{31}^{2}\right|+\Delta m_{21}^{2}}, m_{3}\right)
$$

and $m_{v, \min }=m_{3}$ for IH. In addition, the neutrino mass spectrum is trivial for $\mathrm{DH}$, namely

$m_{1}=m_{2}=m_{3}=m_{v, \min }$. 
Thus we can constrain the sum of the neutrino mass $\sum m_{v}$ via referring to the above three $v \Lambda \mathrm{CDM}$ model. It should be noted that there are lower cut-off values of $\sum m_{v}$, which are $0.06 \mathrm{eV}$ for $\mathrm{NH}$ and $0.10 \mathrm{eV}$ for $\mathrm{IH}$, respectively.

\section{Results}

In this section, we represent the constraints on the neutrino sectors by updating cosmological data. To be specific, we give an updated upper bound on the sum of the neutrino mass $\sum m_{v}$ in Sect. 3.1. In Sect. 3.2, the relativistic degree of freedom $N_{\text {eff }}$ is constrained. We simultaneously constrain $N_{\text {eff }}$ and massive sterile neutrino $m_{v, \text { sterile }}^{\text {eff }}$ in Sect. 3.3.

\subsection{Constraints on $\sum m_{v}$}

In this subsection, we refer to two combinations of datasets, namely Planck TT, TE, EE + lowP + BAO and Planck $\mathrm{TT}+$ lowP + lensing + BAO, to constrain the sum of the neutrino mass $\sum m_{v}$ with $\mathrm{NH}, \mathrm{IH}$, and $\mathrm{DH}$, respectively. In the $v \Lambda \mathrm{CDM}$ model, the free cosmological parameters are given by

$\left\{\omega_{b}, \omega_{c}, 100 \theta_{\mathrm{MC}}, \tau, n_{s}, \ln \left(10^{10} A_{s}\right), m_{v, \min }\right\}$

where $m_{v, \min }$ is the minimal eigenvalue of neutrino mass, and the total mass of neutrinos is a derived parameter, i.e. $\sum m_{v}=m_{1}+m_{2}+m_{3}$.

For three hierarchies, our constraints on $\sum m_{v}$ as well as seven free parameters and $\chi^{2}$ can be found in Table 2 .

The likelihood distributions of $\sum m_{v}$ and $m_{v, \min }$ are depicted in Fig. 1.

The dashed lines denote constraints from Planck TT + lowP + lensing + BAO dataset, while the solid lines denote constraints from Planck TT, TE, EE + lowP + BAO dataset. The red, black, and blue lines denote constraints for the $\mathrm{NH}, \mathrm{IH}$, and DH of the neutrino mass spectrum, respectively. The gray dashed lines denote the minimum values for the total mass of the three neutrinos for $\mathrm{NH}$ and $\mathrm{IH}$, respectively.

For the DH, the $95 \%$ C.L. upper limit on the total mass of three active neutrinos is $\sum m_{v}<0.15 \mathrm{eV}$ for the data combination of Planck TT, TE, EE + lowP + BAO. The best-fit likelihoods is $\chi_{\mathrm{DH}}^{2}=12950.94$. Compared to Planck 2015 constraint $\sum m_{v}<0.17 \mathrm{eV}$ in [17] from the Planck TT, TE, $\mathrm{EE}+$ lowP + BAO data where the BOSS DR11 CMASS and LOWZ data are used, there is about $10 \%$ improvement on the uncertainty. The reason is that the total volume probed in BOSS DR12 has a $10 \%$ increment and the experimental uncertainties are improved correspondingly. A conservative estimate is $\sum m_{v}<0.23 \mathrm{eV}$ with the best-fit likelihood $\chi_{\mathrm{DH}}^{2}=11284.34$ from the data combination of Planck $\mathrm{TT}+$ lowP + lensing $+\mathrm{BAO}$.

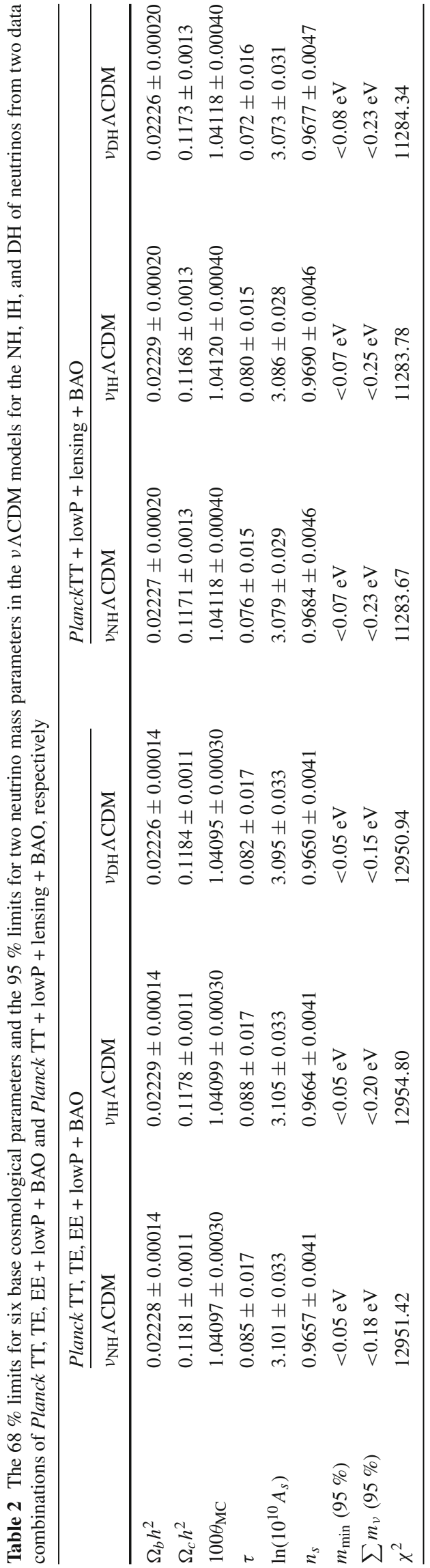



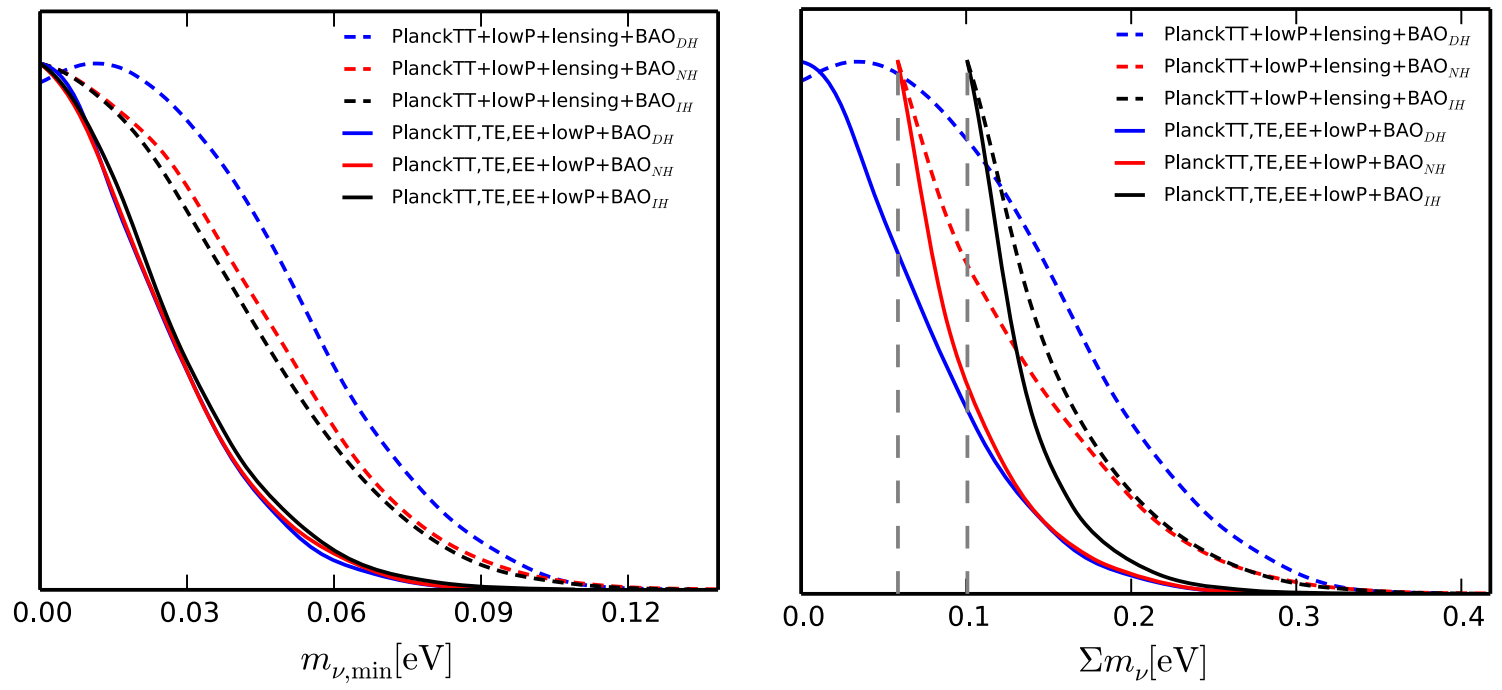

Fig. 1 The likelihood distributions of $m_{v, \min }$ and $\sum m_{v}$ for the $\mathrm{NH}$, IH, and $\mathrm{DH}$ of neutrinos in the $v \Lambda \mathrm{CDM}$ models from two data combinations of Planck TT, TE, EE + lowP + BAO and Planck TT + lowP + lensing + BAO, respectively

For the NH, our constraint on $\sum m_{v}$ is given by $\sum m_{v}<$ $0.18 \mathrm{eV}$ at $95 \%$ C.L. from Planck TT, TE, EE + lowP + BAO dataset. It is around $20 \%$ looser than the above constraint for the $\mathrm{DH}$ from the same dataset. The best-fit likelihood for $\mathrm{NH}$ is $\chi_{\mathrm{NH}}^{2}=12951.42$, which is slightly larger than that for $\mathrm{DH}$. On the other hand, our constraint becomes $\sum m_{v}<0.23$ $\mathrm{eV}$ at $95 \%$ C.L. for Planck TT + lowP + lensing + BAO dataset, and the best-fit likelihood is $\chi_{\mathrm{NH}}^{2}=11283.67$. This constraint is similar to the constraint for $\mathrm{DH}$ by Planck $\mathrm{TT}+$ lowP + lensing + BAO dataset since this constraint is too loose to be sensitive to the neutrino mass hierarchy.

For the $\mathrm{IH}$, our constraint on $\sum m_{v}$ is given by $\sum m_{v}<$ $0.20 \mathrm{eV}$ at $95 \%$ C.L. from Planck TT, TE, EE + lowP + BAO dataset. It is more than $30 \%$ larger than that for the $\mathrm{DH}$ and about $10 \%$ larger than that for the $\mathrm{NH}$ from the same dataset. The best-fit likelihoods is $\chi_{\mathrm{IH}}^{2}=12954.80$ which is larger than that for NH by $\Delta \chi^{2}=\chi_{\mathrm{IH}}^{2}-\chi_{\mathrm{NH}}^{2} \simeq 3$.4. It implies that the current data slightly prefers a normal hierarchy. On the other hand, our constraint becomes $\sum m_{v}<0.25 \mathrm{eV}$ at $95 \%$ C.L. for Planck TT + lowP + lensing + BAO dataset.

\subsection{Constraints on $N_{\text {eff }}$}

The total energy density of radiation in the Universe is given by

$\rho=\left[1+N_{\mathrm{eff}} \frac{7}{8}\left(\frac{4}{11}\right)^{4 / 3}\right] \rho_{\gamma}$

where $\rho_{\gamma}$ is the energy density of CMB photons and $N_{\text {eff }}=$ 3.046 for counting the standard model neutrinos. $N_{\text {eff }}>$ 3.046 will indicate that there are some unknown relativistic degrees of freedom in the Universe.
In this subsection, we use two data combinations of Planck TT, TE, EE + lowP + BAO and Planck TT + lowP + lensing + BAO to constrain $N_{\text {eff }}$ or equivalently the additional relativistic degree of freedom $\Delta N_{\text {eff }}=N_{\text {eff }}-3.046$ in the base $\Lambda \mathrm{CDM}+N_{\text {eff }}$ model. The free parameters include six base parameters and $N_{\text {eff }}$, while we fix $\sum m_{v}=0.06 \mathrm{eV}$ with two massless and one massive active neutrinos. Our constraints on $N_{\text {eff }}$ can be found in Table 3, where we also list constraints on other free parameters.

Our results are well consistent with the standard prediction $N_{\text {eff }}=3.046$. The constraints on the effective number of relativistic degrees of freedom are $N_{\text {eff }}=3.06 \pm 0.36$ and $N_{\text {eff }}=3.10_{-0.44}^{+0.45}$ at $95 \%$ C.L. from Planck TT, TE, $\mathrm{EE}+$ lowP + BAO and Planck $\mathrm{TT}+$ low + lensing + BAO, respectively. $\Delta N_{\text {eff }}=1$, for example a fully thermalized sterile neutrino, is excluded at higher than $5 \sigma$ level by Planck TT, TE, EE + lowP + BAO data and at $4 \sigma$ level by Planck $\mathrm{TT}+$ lowP + lensing + BAO data. A thermalized massless boson decoupled in the range $0.5 \mathrm{MeV}<T<100 \mathrm{MeV}$ predicts $\Delta N_{\text {eff }}=4 / 7 \simeq 0.57$ [36], which is disfavored at more than $95 \%$ C.L. by these two datasets. If it decoupled at $T>100 \mathrm{MeV}, \Delta N_{\mathrm{eff}} \simeq 0.39$ [36], which is slightly disfavored by Planck TT, TE, EE + lowP + BAO data but slightly favored by Planck TT + lowP + lensing + BAO data.

\subsection{Simultaneous constraints on $N_{\text {eff }}$ and $m_{\nu \text {,sterile }}^{\text {eff }}$}

We can also consider extra one massive sterile neutrino whose effective mass is parametrized by $m_{v \text {,sterile }}^{\text {eff }} \equiv\left(94.1 \Omega_{v \text {,sterile }}\right.$ $h^{2}$ ) eV. Assuming the sterile neutrino to be thermally distributed with an arbitrary temperature, $m_{v, \text { sterile }}^{\text {eff }}$ is then given by 
Table 3 The $68 \%$ limits for six base cosmological parameters and the $95 \%$ limits for two neutrino parameters in the base $\Lambda \mathrm{CDM}+N_{\text {eff }}$ and base $\Lambda \mathrm{CDM}+N_{\text {eff }}+m_{\nu \text {, sterile }}^{\text {eff }}$ models from two data combinations of Planck TT, TE, EE + lowP + BAO and Planck TT + lowP + lensing $+\mathrm{BAO}$, respectively

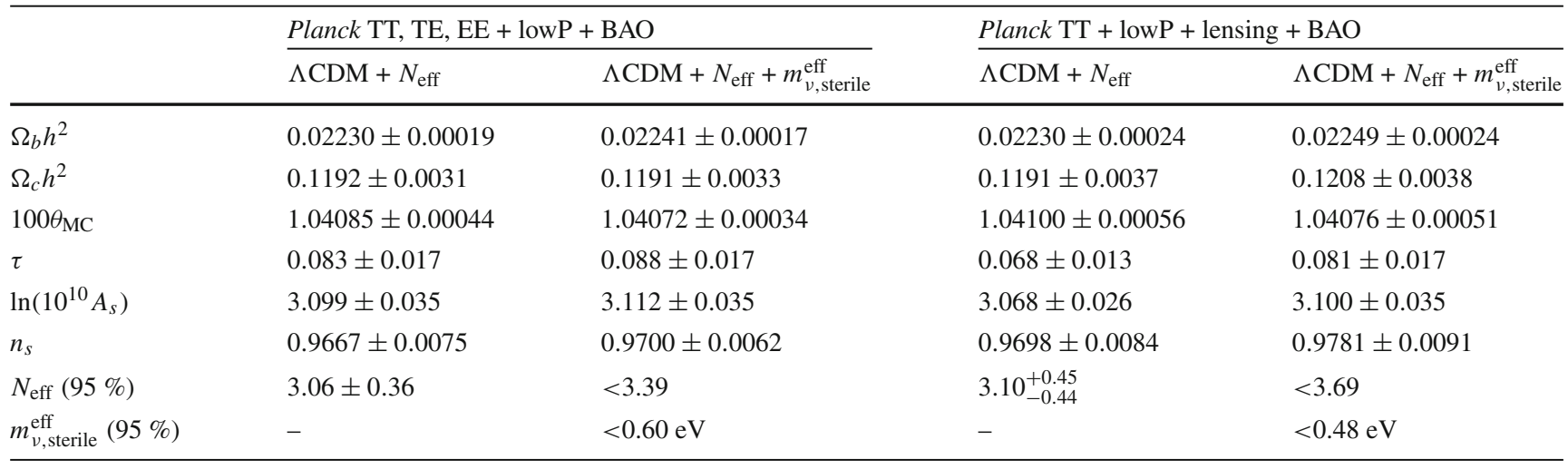

$m_{v, \text { sterile }}^{\text {eff }}=\left(\Delta N_{\text {eff }}\right)^{3 / 4} m_{\text {sterile }}^{\text {thermal }}$,

where $m_{\text {sterile }}^{\text {thermal }}$ denotes the true mass. Here we consider the base $\Lambda \mathrm{CDM}+N_{\text {eff }}+m_{v \text {, sterile }}^{\text {eff }}$ model, in which $m_{\text {sterile }}^{\text {thermal }}$ is a free parameter with a prior $m_{\text {sterile }}^{\text {thermal }}<10 \mathrm{eV}$ and $N_{\text {eff }}$ has a flat prior with $N_{\text {eff }}>3.046$.

Our simultaneous constraints on $N_{\text {eff }}$ and $m_{v \text {,sterile }}^{\text {eff }}$ can be found in Table 3. From Planck TT, TE, EE + lowP + BAO data, we obtain constraints to be $N_{\text {eff }}<3.39$ and $m_{v \text {, sterile }}^{\text {eff }}<$ $0.60 \mathrm{eV}$ at $95 \%$ C.L.. From Planck TT + lowP + lensing + BAO data, we obtain $N_{\text {eff }}<3.69$ and $m_{v \text {,sterile }}^{\text {eff }}<0.48$ eV at $95 \%$ C.L., which are similar to Planck 2015 results in [17]. $\Delta N_{\text {eff }}=1$ can be excluded at much more than $95 \%$ C.L.. One should note that the upper tail of $m_{v \text {, sterile }}^{\text {eff }}$ is closely related to the high physical masses close to the prior cutoff.

\section{Conclusions}

In this paper, we first updated cosmological constraints on the total mass of three degenerate active neutrinos by updating BOSS DR11 to DR12 of CMASS and LOWZ samples. Then we considered the mass splitting between three neutrinos and gave cosmological constraints on the neutrino mass hierarchy. When the Planck TT, TE, EE + lowP + BAO combination is updated, our constraint $\sum m_{v}<0.15 \mathrm{eV}$ at $95 \%$ C.L. is improved by about $10 \%$ for the DH, comparing to Planck 2015 constraint $\sum m_{v}<0.17 \mathrm{eV}$ at $95 \%$ C.L. [17]. When the mass splitting is considered, we get $95 \%$ C.L. upper limits $\sum m_{v}<0.18 \mathrm{eV}$ for the $\mathrm{NH}$ and $\sum m_{v}<0.20 \mathrm{eV}$ for the IH. For the $\mathrm{NH}$ (or the IH) and the $\mathrm{DH}$, there is about $20 \%$ (or $33 \%$ ) difference between their upper limits on the summed neutrino mass. Thus it is meaningful to take into consideration the data of the neutrino mass splitting obtained from the experimental particle physics. Although the current cosmological data may be not good enough to distinguish different neutrino mass hierarchies, the normal hierarchy is slightly preferred by $\Delta \chi^{2} \simeq-3.4$ compared to the inverted hierarchy in our paper. That is to say, the current cosmological data has already put some pressure on the inverted hierarchy. Future precise observations might have the potential to determine the neutrino mass and mass hierarchy completely [37-52].

There are various tight constraints on $\sum m_{v}$ in the literature. For instance, the combination of Lyman- $\alpha$ absorption in the distant quasar spectra, $\mathrm{BAO}$, and Planck CMB data gave a constraint $\sum m_{v}<0.12 \mathrm{eV}$ at $95 \%$ C.L. in [15]. The combination of SDSS DR7 Luminous Red Galaxies (LRG), BAO, and Planck CMB data gave an upper bound $\sum m_{v}<0.11 \mathrm{eV}$ at $95 \%$ C.L. in [16]. Both constraints, close to the lower cutoff values of $0.10 \mathrm{eV}$ for the $\mathrm{IH}$, are tighter than ours, obtained in this paper. Thus it is interesting to include the observational datasets regarding the matter power spectrum in our exploration, besides the lensed-CMB and BAO data. We will address these considerations in our future work. There are also some papers where the neutrino mass hierarchy is taken into consideration. For example, the combination of the WiggleZ Dark Energy Survey power spectrum, BAO, and Planck $\mathrm{CMB}$ data gave similar $\chi^{2} \approx 10261$ for the $\mathrm{DH}, \mathrm{NH}$, and $\mathrm{IH}$ in [9]. By contrast, our data combination favors the NH by $\Delta \chi^{2} \simeq-3.4$ compared to the IH.

In addition, we also updated the constraints on the relativistic degree of freedom and massive sterile neutrinos. Our results are similar to Planck 2015 constraints in [17]. We found no significant evidence for additional relativistic degree of freedom and fully thermalized massive sterile neutrinos by using current datasets in this paper. Nevertheless, a significant density of additional radiations is still allowed on considering the uncertainties of the data.

Acknowledgments We acknowledge the use of HPC Cluster of SKLTP/ITP-CAS. This work is supported by Top-Notch Young Talents Program of China and grants from NSFC (Grant Nos. 11322545, 
11335012 and 11575271). QGH would also like to thank the participants of the advanced workshop "Dark Energy and Fundamental Theory" supported by the Special Fund for Theoretical Physics from the National Natural Science Foundations of China (Grant No. 11447613) for useful conversation.

Open Access This article is distributed under the terms of the Creative Commons Attribution 4.0 International License (http://creativecomm ons.org/licenses/by/4.0/), which permits unrestricted use, distribution, and reproduction in any medium, provided you give appropriate credit to the original author(s) and the source, provide a link to the Creative Commons license, and indicate if changes were made. Funded by SCOAP ${ }^{3}$.

\section{References}

1. J. Lesgourgues, S. Pastor, Phys. Rep. 429, 307 (2006). arXiv:astro-ph/0603494

2. K.A. Olive et al., Particle Data Group, Chin. Phys. C 38, 090001 (2014)

3. W. Hu, D.J. Eisenstein, M. Tegmark, Phys. Rev. Lett. 80, 5255 (1998). arXiv:astro-ph/9712057

4. W. Hu, S. Dodelson, Ann. Rev. Astron. Astrophys. 40, 171 (2002). arXiv:astro-ph/0110414

5. E. Komatsu et al., WMAP Collaboration, Astrophys. J. Suppl. 180, 330 (2009). arXiv:0803.0547 [astro-ph]

6. R. Jimenez, T. Kitching, C. Pena-Garay, L. Verde, JCAP 1005, 035 (2010). arXiv:1003.5918 [astro-ph.CO]

7. B.A. Reid, L. Verde, R. Jimenez, O. Mena, JCAP 1001, 003 (2010). arXiv:0910.0008 [astro-ph.CO]

8. S.A. Thomas, F.B. Abdalla, O. Lahav, Phys. Rev. Lett. 105, 031301 (2010). arXiv:0911.5291 [astro-ph.CO]

9. S. Riemer-Sorensen, D. Parkinson, T.M. Davis, Phys. Rev. D 89, 103505 (2014). arXiv:1306.4153 [astro-ph.CO]

10. M.E.C. Swanson, W.J. Percival, O. Lahav, Mon. Not. R. Astron. Soc. 409, 1100 (2010). arXiv:1006.2825 [astro-ph.CO]

11. E. Di Valentino, E. Giusarma, M. Lattanzi, O. Mena, A. Melchiorri, J. Silk, Phys. Lett. B 752, 182 (2016). arXiv:1507.08665 [astroph.CO]

12. E. Di Valentino, E. Giusarma, O. Mena, A. Melchiorri, J. Silk, Phys. Rev. D 93(8), 083527 (2016). arXiv:1511.00975 [astro-ph.CO]

13. G. Rossi, C. Yche, N. Palanque-Delabrouille, J. Lesgourgues, Phys. Rev. D 92(6), 063505 (2015). arXiv:1412.6763 [astro-ph.CO]

14. Z. Hou et al., Astrophys. J. 782, 74 (2014). arXiv:1212.6267 [astroph.CO]

15. N. Palanque-Delabrouille et al., JCAP 1511(11), 011 (2015). arXiv: 1506.05976 [astro-ph.CO]

16. A.J. Cuesta, V. Niro, L. Verde, Phys. Dark Univ. 13, 77-86 (2016). arXiv:1511.05983 [astro-ph.CO]

17. Planck Collaboration, P.A.R. Ade et al., A\&A (2016). arXiv: 1502.01589 [astro-ph.CO]

18. M. Gerbino, M. Lattanzi, A. Melchiorri, Phys. Rev. D 93, 033001 (2016). arXiv:1507.08614 [hep-ph]

19. J.F. Zhang, M.M. Zhao, Y.H. Li, X. Zhang, JCAP 1504, 038 (2015). arXiv:1502.04028 [astro-ph.CO]

20. X. Zhang, Phys. Rev. D 93, 083011 (2016). arXiv:1511.02651 [astro-ph.CO]

21. Planck Collaboration, P.A.R. Ade et al., A\&A (2015). doi:10.1051/ 0004-6361/201525941. arXiv:1502.01591 [astro-ph.CO]

22. F. Beutler et al., Mon. Not. R. Astron. Soc. 416, 3017 (2011). arXiv:1106.3366 [astro-ph.CO]

23. A.J. Ross, L. Samushia, C. Howlett, W.J. Percival, A. Burden, M. Manera, Mon. Not. R. Astron. Soc. 449(1), 835 (2015). arXiv:1409.3242 [astro-ph.CO]
24. L. Anderson et al., BOSS Collaboration, Mon. Not. R. Astron. Soc. 441(1), 24 (2014). arXiv:1312.4877 [astro-ph.CO]

25. A.J. Cuesta et al., MNRAS 457(2), 1770-1785 (2016). arXiv:1509.06371 [astro-ph.CO]

26. H. Gil-Marín et al., MNRAS 460(4), 4210-4219 (2016). arXiv:1509.06373 [astro-ph.CO]

27. Planck Collaboration, N. Aghanim et al., A\&A (2016). doi:10. 1051/0004-6361/201526926. arXiv:1507.02704 [astro-ph.CO]

28. C. Heymans et al., Mon. Not. R. Astron. Soc. 427, 146 (2012). arXiv:1210.0032 [astro-ph.CO]

29. T. Erben et al., Mon. Not. R. Astron. Soc. 433, 2545 (2013). arXiv: 1210.8156 [astro-ph.CO]

30. L. Samushia et al., Mon. Not. R. Astron. Soc. 439(4), 3504 (2014). arXiv:1312.4899 [astro-ph.CO]

31. P.A.R. Ade et al., Planck Collaboration, Astron. Astrophys. 571, A20 (2014). arXiv:1303.5080 [astro-ph.CO]

32. A.G. Riess et al., Astrophys. J. 730, 119 (2011). arXiv:1103.2976 [astro-ph.CO] [Astrophys. J. 732, 129 (2011)]

33. W.L. Freedman, B.F. Madore, V. Scowcroft, C. Burns, A. Monson, S.E. Persson, M. Seibert, J. Rigby, Astrophys. J. 758, 24 (2012). arXiv:1208.3281 [astro-ph.CO]

34. G. Efstathiou, Mon. Not. R. Astron. Soc. 440(2), 1138 (2014). arXiv:1311.3461 [astro-ph.CO]

35. A. Lewis, S. Bridle, Phys. Rev. D 66, 103511 (2002). arXiv:astro-ph/0205436

36. S. Weinberg, Phys. Rev. Lett. 110(24), 241301 (2013). arXiv:1305.1971 [astro-ph.CO]

37. C. Carbone, L. Verde, Y. Wang, A. Cimatti, JCAP 1103, 030 (2011). arXiv:1012.2868 [astro-ph.CO]

38. Y.Y.Y. Wong, Ann. Rev. Nucl. Part. Sci. 61, 69 (2011). arXiv:1111.1436 [astro-ph.CO]

39. A.C. Hall, A. Challinor, Mon. Not. R. Astron. Soc. 425, 1170 (2012). arXiv:1205.6172 [astro-ph.CO]

40. J. Hamann, S. Hannestad, Y.Y.Y. Wong, JCAP 1211, 052 (2012). arXiv:1209.1043 [astro-ph.CO]

41. B. Audren, J. Lesgourgues, S. Bird, M.G. Haehnelt, M. Viel, JCAP 1301, 026 (2013). arXiv:1210.2194 [astro-ph.CO]

42. A. Font-Ribera, P. McDonald, N. Mostek, B.A. Reid, H.J. Seo, A. Slosar, JCAP 1405, 023 (2014). arXiv:1308.4164 [astro-ph.CO]

43. K.N. Abazajian et al., Topical Conveners: K.N. Abazajian, J.E. Carlstrom, A.T. Lee Collaboration, Astropart. Phys. 63, 66 (2015). arXiv: 1309.5383 [astro-ph.CO]

44. W.L.K. Wu, J. Errard, C. Dvorkin, C.L. Kuo, A.T. Lee, P. McDonald, A. Slosar, O. Zahn, Astrophys. J. 788, 138 (2014). arXiv: 1402.4108 [astro-ph.CO]

45. E.M. Mueller, F. de Bernardis, R. Bean, M.D. Niemack, Phys. Rev. D 92(6), 063501 (2015). arXiv:1412.0592 [astro-ph.CO]

46. Z. Pan, L. Knox, MNRAS 454(3), 3200-3206 (2015). arXiv:1506.07493 [astro-ph.CO]

47. F. Villaescusa-Navarro, P. Bull, M. Viel, Astrophys. J. 814(2), 146 (2015). arXiv:1507.05102 [astro-ph.CO]

48. J. Errard, S.M. Feeney, H.V. Peiris, A.H. Jaffe, J. Cosmol. Austropart. Phys. 2016(03), 052 (2016). arXiv:1509.06770 [astroph.CO]

49. R. Allison, P. Caucal, E. Calabrese, J. Dunkley, T. Louis, Phys. Rev. D 92, 123535 (2015). arXiv:1509.07471 [astro-ph.CO]

50. A. Liu, J.R. Pritchard, R. Allison, A.R. Parsons, U. Seljak, B.D. Sherwin, Phys. Rev. D 93, 043013 (2016). arXiv: 1509.08463 [astro-ph.CO]

51. Y. Oyama, K. Kohri, M. Hazumi, J. Cosmol. Austropart. Phys. 2016(02), 008 (2016). arXiv:1510.03806 [astro-ph.CO]

52. G.B. Zhao et al., MNRAS 457(3), 2377-2390 (2016). arXiv:1510.08216 [astro-ph.CO] 